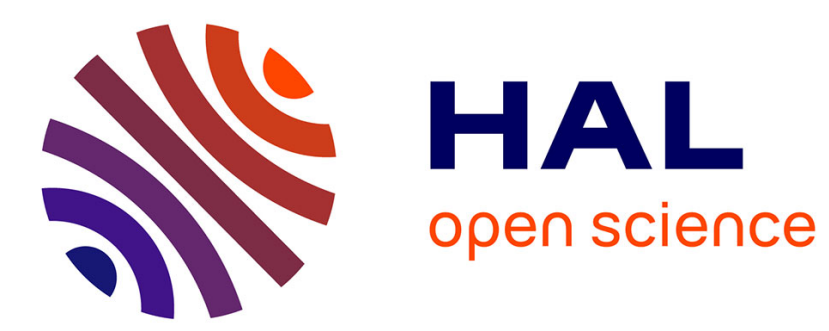

\title{
Contribution au dosage de l'oxygène dans les pellicules superficielles par irradiation avec des tritons de $3 \mathrm{Mev}$
}

Jean-Noël Barrandon, Ph. Albert

\section{To cite this version:}

Jean-Noël Barrandon, Ph. Albert. Contribution au dosage de l'oxygène dans les pellicules superficielles par irradiation avec des tritons de 3 Mev. Revue de Physique Appliquée, 1968, 3 (2), pp.111-115. 10.1051/rphysap:0196800302011100 . jpa-00242832

\section{HAL Id: jpa-00242832 https://hal.science/jpa-00242832}

Submitted on 1 Jan 1968

HAL is a multi-disciplinary open access archive for the deposit and dissemination of scientific research documents, whether they are published or not. The documents may come from teaching and research institutions in France or abroad, or from public or private research centers.
L'archive ouverte pluridisciplinaire HAL, est destinée au dépôt et à la diffusion de documents scientifiques de niveau recherche, publiés ou non, émanant des établissements d'enseignement et de recherche français ou étrangers, des laboratoires publics ou privés. 


\title{
GONTRIBUTION AU DOSAGE DE L'OXYGÈNE DANS LES PELLIGULES SUPERFIGIELLES PAR IRRADIATION AVEG DES TRITONS DE $3 \mathrm{MeV}$
}

\author{
Par J. N. BARRANDON et Ph. ALBERT, \\ Laboratoire d'Analyse par Activation du Centre d'Études de Chimie Métallurgique de Vitry-sur-Seine (France).
}

(Reçu le 23 septembre 196\%.)

\begin{abstract}
Résumé. - On a déterminé la courbe d'excitation de la réaction ${ }^{16} \mathrm{O}(\mathrm{T}, \mathrm{n}){ }^{18} \mathrm{~F}$ pour une énergie des tritons variant de 0,5 à $3,0 \mathrm{MeV}$. Nous exposons, d'autre part, une méthode de mesure d'épaisseur de pellicule d'oxyde à la surface des métaux par irradiation dans les tritons.

Abstract. - The excitation function of the reaction ${ }^{16} \mathrm{O}(\mathrm{T}, \mathrm{n})^{18} \mathrm{~F}$ has been determined when the triton energy varies from 0.5 to $3.0 \mathrm{MeV}$. Additionnally, we present a method for the determination of metal oxide film thickness, by triton irradiation.
\end{abstract}

Introduction. - Smales [1] avait montré dès 1949 la possibilité de doser l'oxygène par les tritons grâce à la réaction :

$$
{ }_{8}^{16} \mathrm{O}(\mathrm{T}, \mathrm{n}){ }_{9}^{18} \mathrm{~F}^{*} \quad(Q=+1,280 \mathrm{MeV}) .
$$

De nombreuses expériences [2] et [3] ont été faites à l'aide d'un faisceau de tritons obtenu par la réaction :

$$
{ }_{8}^{6} \mathrm{Li}(\mathrm{n}, \alpha) \mathrm{T} \text {. }
$$

Cette réaction dont la section efficace pour des neutrons thermiques est de 910 barns produit des tritons d'une énergie maximale égale à $2,74 \mathrm{MeV}$.

Cette méthode de production de tritons présente plusieurs inconvénients :

1) Les réactions produites par le flux de neutrons thermiques empêchent un dosage non destructif de l'oxygène dans la plupart des matériaux;

2) Le faisceau de tritons n'est pas monoénergétique mais présente un spectre d'énergie. Tout dosage dans la masse d'un échantillon massif est donc très difficile;

3) Il n'est pas possible de doser l'oxygène de surfaces parfaitement propres. En effet, le contact nécessaire avec le lithium ou un de ses sels polluera pendant l'irradiation cette surface.

C'est pourquoi nous avons étudié la possibilité de doser l'oxygène à la surface des métaux en accélérant les tritons dans un Van de Graaff.

I. Dispositif expérimental. - Ges expériences ont été effectuées au Van de Graaff vertical de $3 \mathrm{MeV}$, du Centre de Recherches Nucléaires de Strasbourg. La bouteille alimentant la source d'ions [4] contient 7 cc de tritium dilué dans de l'hélium, dans un rap- port 3/1 000 en volume. Dans ces conditions, l'accélérateur fournit un faisceau de tritons analysé et diaphragmé d'une intensité de l'ordre de $0,05 \mu \mathrm{A}$ pendant 160 heures.

La mesure du courant et l'intégration de celui-ci ont été faites à l'aide d'un intégrateur : "Elcor ».

Nous disposions, d'autre part, d'une installation de spectrométrie $\gamma$.

II. Courbe d'excitation de la réaction : ${ }^{16} \mathrm{O}(\mathrm{T}, \mathrm{n}){ }^{18} \mathrm{~F}^{*}$. - Dans une première série d'expériences, nous avons déterminé la courbe d'excitation de la réaction ${ }_{8}^{16} \mathrm{O}(\mathrm{T}, \mathrm{n}){ }_{9}^{18} \mathrm{~F}^{*}$ pour une énergie des tritons variant de 0 à $3 \mathrm{MeV}$.

Pour cela, nous avons irradié des empilements de disques de mica (étalon oxygène) dont les épaisseurs s'échelonnaient entre 3 et 10 microns. L'activité du fluor 18, obtenu par réaction des tritons sur l'oxygène contenu dans le mica (48\%), est mesurée par les $\gamma$ de $511 \mathrm{keV}$ émis par annihilation du $\beta^{+}$. Ayant calculé le parcours des tritons dans le mica à l'aide des tables [5], nous avons obtenu une courbe donnant l'activité en fluor 18 en fonction de l'énergie des tritons.

La perte d'énergie des tritons à travers les différents micas varie de 150 à $550 \mathrm{keV}$ suivant l'énergie incidente du faisceau de tritons et l'épaisseur des disques de mica.

La longueur des barres d'erreur horizontales correspond à la valeur de cette perte d'énergie des tritons à travers chaque mica.

Ne pouvant mesurer la section efficace absolue, nous avons pris, pour étalonner notre courbe de section efficace, un point de la courbe de Jarmie [6]. Cette 


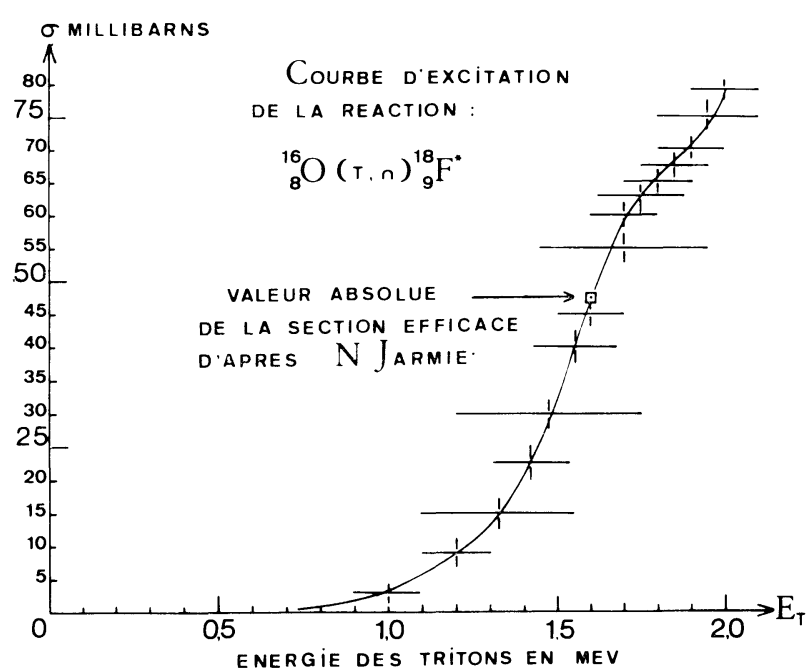

FIG. 1. - Courbe d'excitation de la réaction ${ }^{16} \mathrm{O}(\mathrm{T}, \mathrm{n}){ }^{18} \mathrm{~F}$ pour des tritons d'une énergie de 0,5 à $2 \mathrm{MeV}$.

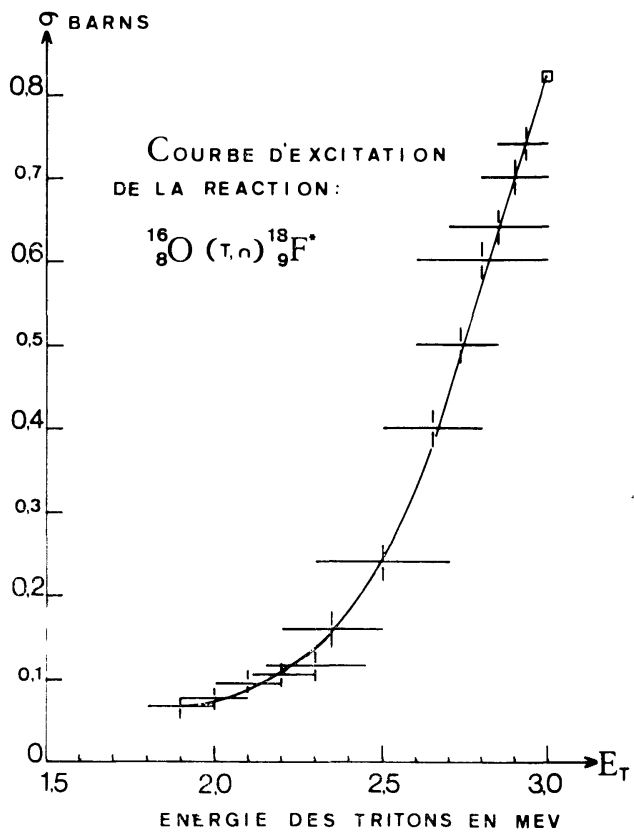

FIG. 2. - Courbe d'excitation de la réaction ${ }^{16} \mathrm{O}(\mathrm{T}, \mathrm{n}){ }^{18} \mathrm{~F}$ pour des tritons d'une énergie de 1,9 à $3 \mathrm{MeV}$.

valeur correspondant à une énergie des tritons de $1,6 \mathrm{MeV}$ est indiquée sur la courbe d'excitation ( fig. 1).

Nous voyons ( $f i g .2$ ) que la section efficace de la réaction ${ }_{8}^{16} \mathrm{O}(\mathrm{T}, \mathrm{n}){ }_{9}^{18} \mathrm{~F}^{*}$ est égale à $0,820 \pm 0,04$ barn pour une énergie de $3 \mathrm{MeV}$.

Nous remarquons ( $f g .2$ ) que la section efficace de la réaction croît fortement à partir d'une valeur de l'énergie des tritons correspondant à la valeur de la barrière de potentiel coulombienne de la réaction $(1,80 \mathrm{MeV})$.
III. Recul du fluor. - L'énergie de recul du ${ }^{18} \mathrm{~F}$ est obtenue en appliquant les lois de conservation de l'énergie et des moments à la réaction ${ }^{16} \mathrm{O}(\mathrm{T}, \mathrm{n})^{18} \mathrm{~F}^{*}$. Cette énergie pour l'angle $\theta=0^{\circ}$ est donnée par la courbe ( fig. 3).

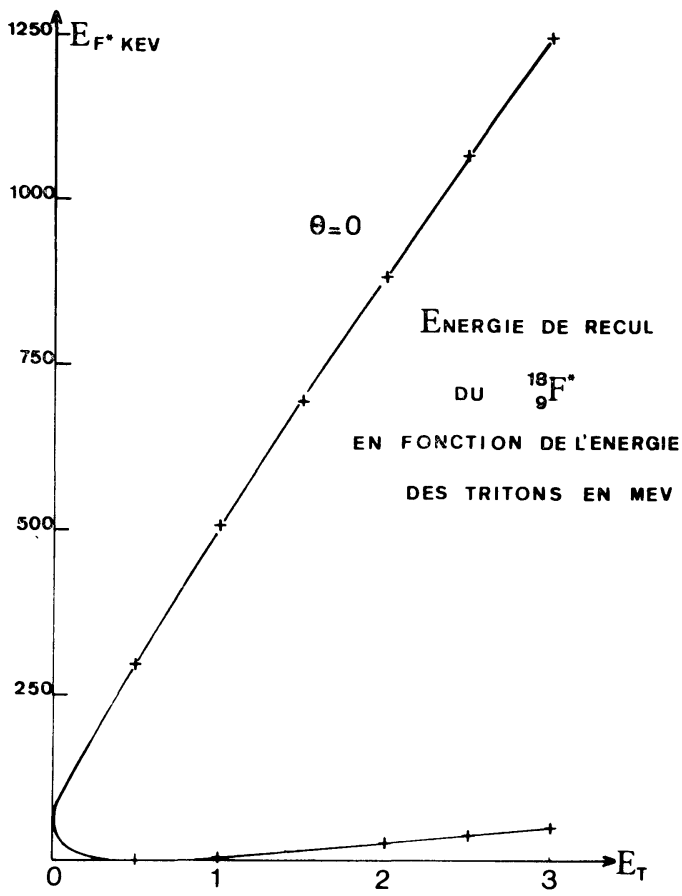

FIG. 3. - Énergie de recul du fluor 18 pour l'angle $\theta=0^{\circ}$ : - courbe supérieure déplacement vers l'avant, - courbe inférieure déplacement vers l'arrière.

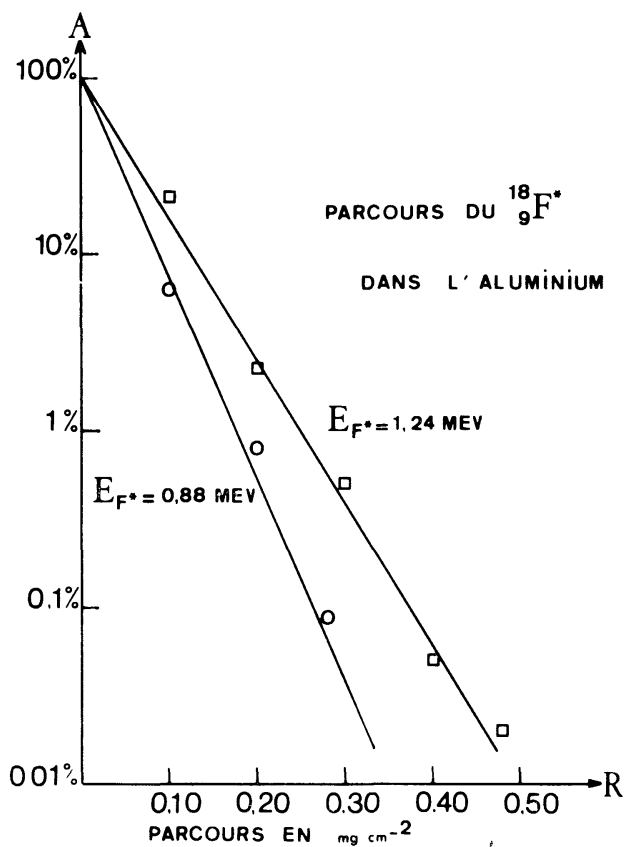

Frg. 4. - Parcours des noyaux de fluor 18 dans l'aluminium pour des énergies de recul de $1,24 \mathrm{MeV}$ et $0,88 \mathrm{MeV}$. 
Pour des tritons de 2 et $3 \mathrm{MeV}$, les énergies de recul $\mathrm{du}$ fluor 18 sont respectivement de $0,88 \mathrm{MeV}$ et $1,24 \mathrm{MeV}$ vers l'avant pour l'angle $\theta=0^{\circ}$.

A l'aide de fines feuilles d'aluminium $\left(0,10 \mathrm{mg} / \mathrm{cm}^{2}\right)$, nous avons pu déterminer le parcours des noyaux de ${ }^{18} \mathrm{~F}$ dans l'aluminium.

Pour une énergie de recul du ${ }^{18} \mathrm{~F}$ de $1,24 \mathrm{MeV}$, le parcours dans l'aluminium est de $0,45 \pm 0,04 \mathrm{mg} / \mathrm{cm}^{2}$; pour une énergie de $0,88 \mathrm{MeV}$, il est de $0,35 \pm$ $0,03 \mathrm{mg} / \mathrm{cm}^{2}$ (fig. 4).

IV. Etalonnage du dosage de l'oxygène contenu dans une pellicule mince à la surface d'un échantillon. - Prenons comme exemple la mesure de l'épaisseur d'une pellicule d'oxyde se trouvant à la surface d'un métal très pur.

Soit un étalon dont la pellicule d'oxyde déposée à la surface du métal a une épaisseur connue $x$ microns, et un échantillon ayant une épaisseur $\Delta x$ suffisamment petite $(\leqslant 0,1 \mu)$ pour que la perte d'énergie $\Delta E$ des tritons à travers cette pellicule soit négligeable, c'est-àdire que la section efficace reste constante dans le domaine d'énergie $E_{0}$ et $E_{0}-\Delta E$.

Nous avons :

$$
\begin{aligned}
\sigma_{(E)}=\sigma_{\left(E_{\dot{0}}\right)}+\left(\frac{\mathrm{d} \sigma}{\mathrm{d} E}\right)_{\left(E_{0}\right)} & \Delta E \\
& =\sigma_{\left(E_{0}\right)}\left[1+\left(\frac{\mathrm{d} \log \sigma}{\mathrm{d} E}\right)_{\left(E_{0}\right)} \Delta E\right] .
\end{aligned}
$$

Il faut donc :

$$
\left(\frac{\mathrm{d} \log \sigma}{\mathrm{d} E}\right)_{\left(E_{0}\right)} \Delta E \ll 1 .
$$

Pour cet étalon et cet échantillon, les activités produites pendant l'irradiation seront respectivement : pour l'étalon :

$$
A_{1}=Q_{1} n_{1} \int_{0}^{x} \sigma\left[E_{(x)}\right] \mathrm{d} x
$$

pour l'échantillon :

$$
A_{2}=Q_{2} n_{2} \int_{0}^{\Delta x} \sigma\left[E_{(x)}\right] \mathrm{d} x
$$

$A_{1}$ et $A_{2}=$ activité en fluor 18 dans l'étalon et l'échantillon,

$Q_{1}$ et $Q_{2}=$ quantité de courant de tritons (valeur donnée par l'intégrateur de courant),

$n_{1}$ et $n_{2} \quad=$ nombre d'atomes d'oxygène par milligramme; les oxydes étant de même nature $n_{1}=n_{2}$,

$\sigma\left(E_{0}\right)=\sigma_{0}=$ section efficace de la réaction à l'énergie $E_{0}$ des particules incidentes,

$\sigma\left[E_{(x)}\right]=\sigma_{x}=$ section efficace à la profondeur $x$ de l'échantillon.

$\mathrm{Si}$ on introduit la notion « d'épaisseur équivalente » [7] définie comme suit :

$$
e=\int_{0}^{x} \sigma\left[E_{(x)}\right] \mathrm{d} x / \sigma\left(E_{0}\right)
$$

$e=$ épaisseur fictive dans laquelle se serait répartie uniformément l'activité ( fig. 5).

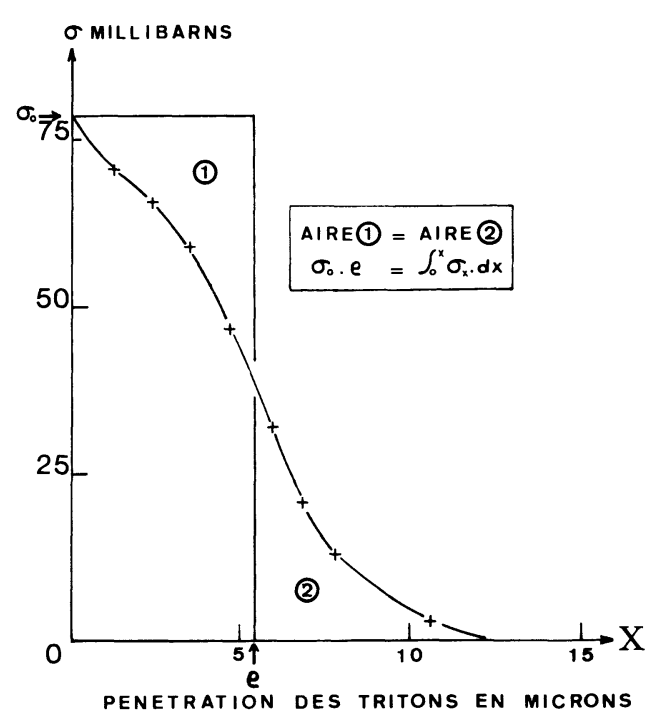

FiG. 5. - Définition de la notion " d'épaisseur équivalente ". Pour des tritons de $2 \mathrm{MeV}$, l'épaisseur équivalente dans la zircone $\left(\mathrm{ZrO}_{2}\right)$ est de 5,5 microns.

On obtient donc :

$$
A_{1}=Q_{1} n_{1} \sigma_{0} e ; \quad A_{2}=Q_{2} n_{2} \sigma_{0} \Delta x
$$

d'où :

$$
\Delta x=e\left[\left(\frac{A_{2}}{A_{1}}\right)\left(\frac{Q_{1}}{Q_{2}}\right)\right] .
$$

V. Choix des conditions expérimentales. - Pour ces mesures d'épaisseur de pellicules d'oxyde superficielles, nous avons choisi une énergie incidente des tritons égale à $2 \mathrm{MeV}$ pour les raisons suivantes :

a) A cette énergie, la section efficace est suffisante pour assurer une bonne sensibilité de détection d'une pellicule d'oxyde même très mince. Par contre, l'oxygène dissous dans le métal sous-jacent ne conduira pas à une activité en ${ }^{18} \mathrm{~F}^{*}$ qui serait gênante pour doser l'oxygène de surface. En effet, premièrement, la teneur en oxygène dissous dans la masse de nos échantillons varie de quelques dizaines à quelques dixièmes de partie par million; deuxièmement, la section efficace diminue rapidement avec la pénétration des tritons dans le métal; troisièmement, pour $2 \mathrm{MeV}$, le parcours des tritons est faible;

b) Pour $2 \mathrm{MeV}$, la variation de section efficace correspondant à une perte d'énergie de $0,1 \mathrm{MeV}$ dans la pellicule d'oxyde de l'échantillon est faible (10\% de la valeur de $\sigma_{0}$, fig. 2).

On peut donc admettre $\sigma_{0}$ constante pour le calcul de l'activité produite par les tritons à travers la pellicule d'oxyde $\Delta x$ qui recouvre la surface de l'échantillon à analyser.

VI. Précision des mesures. - L'erreur relative sur $\Delta x$ sera égale à la somme des erreurs relatives de chacun des termes de la formule donnant $\Delta x$. Les 
erreurs absolues sur les termes $e, A_{1}, A_{2}, n_{1}$ et $n_{2}$ sont facilement chiffrables; par contre, les erreurs absolues sur les mesures de $Q_{1}$ et de $Q_{2}$ sont très difficiles à estimer. Ces erreurs dépendent entre autres des réglages de l'intégrateur de courant et des électrons secondaires émis par la cible. Pour des conditions identiques d'énergie et de mesure de courant, le taux d'électrons secondaires reste constant [8]. En ce qui concerne le réglage de l'intégrateur de courant, on a au maximum $5 \%$ d'erreur entre deux séries d'expériences (réglages différents), mais dans une même série d'expériences (réglages identiques) l'erreur est inférieure à $1 \%$. Nous pensons que la solution qui entraînerait le moins d'erreurs sur le dosage serait de monter dans la chambre à diffusion une cible tournante. Un tel montage permettrait de présenter alternativement sous le faisceau l'échantillon et l'étalon. Le montage d'un tel dispositif est envisagé.

Dans l'état actuel de nos recherches, la mesure de $\Delta x$ peut être connue avec une précision de l'ordre de $10 \%$.

VII. Résultats et discussion. - Nous avons, dans une première série d'expériences, cherché à voir si notre méthode d'étalonnage était précise et reproductible. Ces expériences ont été effectuées à une énergie incidente du faisceau de tritons égale à $2 \mathrm{MeV}$. Les échantillons de zirconium oxydés en surface ont été irradiés sous vide.

La durée de l'irradiation varie entre 3 et 10 minutes

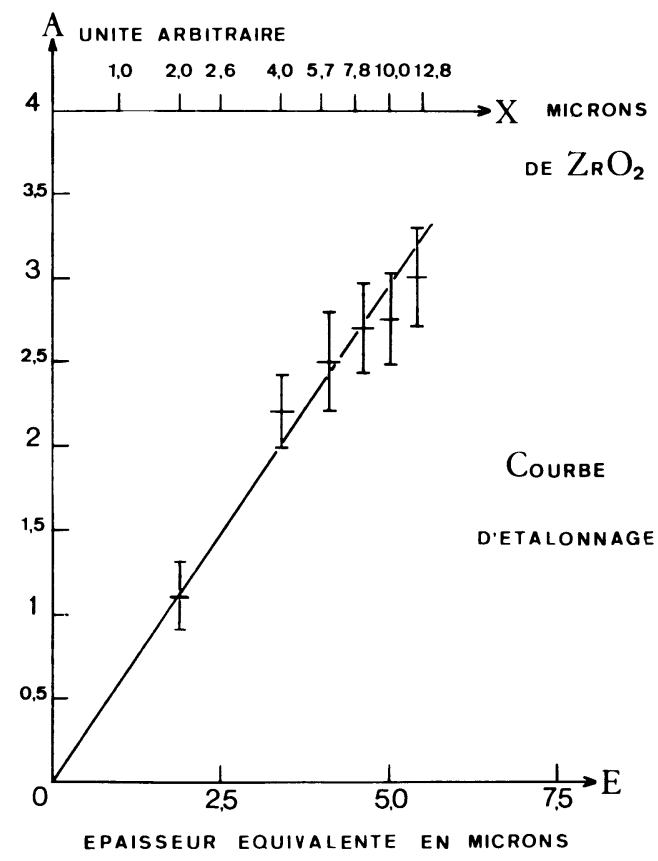

FIG. 6. - Courbe d'étalonnage de la mesure de l'épaisseur de pellicules de $\mathrm{ZrO}_{2}$ recouvrant du zirconium métallique. Cette courbe relie l'activité de fluor 18 à l'épaisseur d'oxyde en microns. selon l'intensité du courant et la sensibilité de détection nécessaire.

La valeur de l'épaisseur de la pellicule d'oxyde de chaque échantillon de zirconium est obtenue par méthode optique avec une très bonne précision $(<5 \%)$.

La courbe d'étalonnage ( fig. 6) nous montre que si l'on tient compte des limites d'erreur (de l'ordre de $10 \%$ ) les points expérimentaux s'alignent sur une droite. D'autre part, pour une même épaisseur d'oxyde, les mesures sont reproductibles (dispersion bien inférieure à $20 \%$ ). Cette série d'expériences nous a permis d'estimer la sensibilité que la méthode peut atteindre. Celle-ci est de l'ordre de la dizaine d'angströms.

La sensibilité de notre méthode nous paraît, dans l'état actuel de nos recherches, moins bonne que celle obtenue par l'utilisation de la réaction ${ }^{16} \mathrm{O}(\mathrm{d}, \mathrm{p})^{17} \mathrm{O}^{*}[9]$. Cette moins bonne sensibilité s'explique par la faible intensité du faisceau de tritons $(0,1 \mu \mathrm{A})$. Mais dans le cas de la mesure de pellicules d'oxyde à la surface de métaux contenant beaucoup d'oxygène dissous en profondeur, le spectre des protons dû à la réaction ${ }^{16} \mathrm{O}(\mathrm{d}, \mathrm{p})^{17} \mathrm{O}^{*}$ présente un pic s'étalant vers les basses énergies. Cette asymétrie de pic est aussi observée pour des pellicules d'oxyde supérieures à $0,1 \mu$. Dans ces deux cas, notre méthode nous semble plus simple et la conduite des calculs plus rapide.

Notre méthode étant une méthode d'activation, il nous est possible de tenir compte de l'oxygène dissous sous la pellicule d'oxyde, soit en ne mesurant que l'activité en fluor 18 produite par la pellicule d'oxyde (dissolution de la pellicule d'oxyde ou du métal sousjacent), soit en soustrayant de l'activité totale l'activité induite par l'oxygène dissous dans le métal (dissolution de la pellicule d'oxyde).

De plus, notre méthode semble présenter un net avantage dans le cas où la détection du pic de la réaction ${ }^{16} \mathrm{O}(\mathrm{d}, \mathrm{p}){ }^{17} \mathrm{O}^{*}$ est masquée par un bruit de fond intense; par exemple, la présence massive d'azote à la surface de l'échantillon ne perturbe pas du tout notre mesure. En effet, pour l'énergie à laquelle nous faisons notre dosage, l'azote ne donne que des isotopes stables ou de période très courte qui ne perturbent pas la mesure de l'activité en fluor 18. Ces isotopes de courte période sont l'azote $16(T=7,35 \mathrm{~s})$ et l'oxygène $15(T=124 \mathrm{~s})$ obtenus par les réactions :

$$
\begin{array}{cc}
{ }^{14} \mathrm{~N}(\mathrm{~T}, \mathrm{p}){ }^{16} \mathrm{~N} & (Q=+4,852 \mathrm{MeV}) \\
{ }^{14} \mathrm{~N}(\mathrm{~T}, 2 \mathrm{n}){ }^{15} \mathrm{O} & (\text { seuil }=1,44 \mathrm{MeV}) .
\end{array}
$$

Conclusions. - En résumé, nous avons décrit des essais préliminaires de dosage par activation très sensible de l'oxygène dans les pellicules superficielles des métaux. Pour définir les meilleures conditions expérimentales, nous avons déterminé la courbe d'excitation de la réaction entre 0,5 et $3 \mathrm{MeV}$. L'emploi 
des tritons accélérés dans un Van de Graaff à une énergie de $2 \mathrm{MeV}$ permet de réaliser ces mesures dans des conditions très propres. Nous avons vérifié que, pour l'aluminium, le zirconium et le nickel, ces mesures peuvent être effectuées sans nécessiter la séparation chimique du fluor 18. L'étalonnage de la méthode montre que le domaine d'utilisation s'étend de la dizaine de microns à la dizaine d'angströms.
Nous tenons à remercier Mme Magnac de nous avoir accueillis dans son Laboratoire et de nous avoir conseillés dans nos recherches.

Nous exprimons notre gratitude à $\mathbf{M}$. Seltz pour les conseils et l'assistance qu'il nous a toujours apportés dans la préparation et la réalisation de nos expériences.

Nous tenons à remercier également l'équipe du Van de Graaff de son aimable collaboration.

\section{BIBLIOGRAPHIE}

[1] Smales, Annal. Rep. Prog. Chem., 1949, 46, 290.

[2] Born (H. J.), Angere. Chem., 1960, 72, 559.

[3] DE GoEIJ (J. J. M.) et Houtman (J. P. W.), Modern Trends in Activation Analysis, 1965.

[4] Magnac-Valeitere (D.), J. Physique Rad., 1956, 17, 449.

[5] Wilitiamson (C.), Boujot (J. P.) et Picard (J.), Rapport C.E.A., no $3042,1966$.

[6] JARmie (N.), Phys. Rep., 1955, 98, Rapport Los Alamos, La 2014, 1956.
[7] Engelmann (Ch.), Thèse C.N.A.M., 1964 ; Rapport C.E.A. no R/2559, 1964.

[8] GorodeTzky (M. S.), BERGDOLT (Mme A. M.), Chevallier (Mme A.), BRES (M.) et ARMBRUSTER (R.), J. Physique, 1963, 24, 374.

[9] Amsel (G.), Thèses, Annales de Physique, 1964, 9, 297.

Amser (G.) et SAmuel (D.), Analytical Chemistry, 1967, 39, 1689. 